\title{
10 -Years Coronary Artery Disease Incidence and its Predictors in Overweight and Obese Adults Using A Competing Risk Model: A Longitudinal Study of Yazd Healthy Heart Cohort (YHHC)
}

\section{Mohsen Askari Shahi}

Center for Health Care Data Modeling, Department Biostatics \& Epidemiology, Health Faculty, Shahid Sadoughi University of Medical Sciences, Yazd, Iran

Seyedeh Mahdieh Namayandeh

Afshar Hospital Clinical Research Development Center, Yazd, Iran

Mahmood Emami Midbody

Yazd Cardiovascular Research Center, Shahid Sadoughi University of Medical Sciences, Yazd, Iran Fatemeh Majidpour ( $\square$ Majidpour.Fa@gmail.com )

Yazd Cardiovascular Research Center, Shahid Sadoughi University of Medical Sciences, Yazd, Iran

\section{Research Article}

Keywords: Competing risk, Coronary Artery Disease, Obesity, Overweight, Yazd Healthy Heart Cohort

Posted Date: January 14th, 2022

DOI: https://doi.org/10.21203/rs.3.rs-1229636/v1

License: (c) (1) This work is licensed under a Creative Commons Attribution 4.0 International License. Read Full License 


\section{Abstract}

We applied competing risk model to identify the predictors for Coronary Artery Disease (CAD) among 866 overweight and obese participants aged 20-74 years using their registered medical records in the first and second phase of Healthy Heart Cohort (YHHC) conducted in Yazd. These participants were free of coronary heart disease in the first phase of study. CAD was considered as the primary event and all other noncardiac deaths were considered as a competing event. The cumulative incidence of any CAD at the 5year and 10 -year follow-ups was approximately $6.8 \%$ and $10.6 \%$, respectively, and approximately $4.6 \%$ and $8.5 \%$, respectively, for all other noncardiac deaths. In both cause-specific and Fine-Gray models of risk factor diabetes type II, hypertriglyceridemia, university level of education (reversely), uric acid, age, systolic blood pressure and female gender (reversely) were associated with the increase risk of CAD. In addition to other traditional cardio metabolic risk factor we found that uric acid increased the risk of CAD in overweight and obese adults. It seems that lifestyle modification can reduce the risk of CAD. Also, high level of education had a protective effect on the risk of CAD. Both cause-specific and fine-gray models predicted similarly 10-years of CAD. The use of competing risk models in the presence of competing events is emphasized when interpreting survival studies.

\section{Key Messages}

- The use of competitive risk models in the presence of a competing cause is emphasized when interpreting survival studies

- UA is a highly significant risk factor for a developed risk of CAD in overweight and obese adults

- High education had a protective effect on decreased risk of CAD in overweight and obese adults.

\section{Introduction}

Coronary artery disease (CAD) and its complications are the main cause of mortality and disability adjusted life years in developed and developing countries(1-3). Half of the annual mortalities in Iran are assigned to themselves(4). Epidemiological evidence has shown that obesity and overweight increase the risk of cardiovascular diseases (CVDs) $(5,6)$. Obesity and overweight have now become a global epidemic(7) and have the greatest effect on CAD (7). by increasing the risk factors for this disease, including diabetes, hypertension, and dyslipidemia(5, 7). According to the results of the Pacific Cohort Collaboration Study on 31000 adults, each unit increase in BMI increases the risk of ischemic heart disease by $9 \%$ (8). The first phase of the Yazd Healthy Heart Cohort reported the prevalence of traditional risk factors for CAD, such as hypertension (25.6\%), diabetes mellitus (11\%), and smoking (13.2\%)(9).

In studies of cardiovascular diseases, the time of disease onset is often considered the primary outcome $(10,11)$. During the follow-up period, patients may not experience the desired event, die until the end of the study or experience several other events before the onset of the disease $(10,12)$. Such events prevent the occurrence of desired events $(10,13)$. or change the probability of their occurrence are called competing risk(10). For example, if a patient dies in CHF, congestive heart failure surgery before the time 
of heart failure occurs(12). death should be considered as censorship(11). This means that at any point in time, these people are at the same risk of CHF as those who have not yet become ill(12). However, these people have died once before(12). and are no longer at risk of disease(11). Standard survival methods, such as Kaplan-Meier analysis to estimate survival rates and Cox regression, were used to investigate the relationship between covariates and disease risk(14). lead to skewed results $(13,15,16)$. and death is a competing risk for heart failure $(12,13,17)$. Two models are commonly used to calculate competing risks: the subdistribution hazard model and the cause-specific hazard model(11). The subdistribution model is used to investigate the effect of covariates on the cumulative incidence function(18).

The cause-specific hazard model is used to investigate the effect of covariates on the cause-specific hazard of outcome, and other causes are censored $(11,13,19)$. The incidence of cardiovascular diseases varies according to cultural and geographical region $(3,19,20)$. Attempts have been made to identify risk factors for CVD(21). and many published studies may fail to consider the method of competing risks(22, 23). This study aimed to calculate the cumulative incidence and determine the predictive risk factors for $C A D$ in the presence of death as a competing risk in overweight and obese individuals in the population of 2074 years in the city of Yazd based on longitudinal data.

\section{Methods}

\subsection{Study Population}

The present study is a retrospective cohort study based on the information of the first and second phases of the Yazd Healthy Heart Cohort study, which is a 15-year cohort study with three phases (each phase five years), and the third phase is underway. A total of 2000 people aged 2074 years in the city of Yazd were included in this study using multistage cluster sampling from fall 2004 to summer 2005. In the first five years, participants were invited annually and examined in terms of demographic factors, anthropometrics, lifestyle, medical history, medications, chest pain questionnaire, electrodiagram of medical history, cardiometabolic risk factors and cardiovascular disease outcomes. In the second phase, the participants were invited again and followed up after five years, and the third phase is currently underway.

\subsection{Clinical and laboratory measurements:}

In the first phase, data were collected through interviews, physical examinations, and laboratory information. Blood pressure and anthropometric factors were collected using physical examination. Height and weight were measured using a digital height and weight scale. Body mass index was calculated based on the classification of the health organization as weight in kilograms divided by height in meters squared(24). Overweight was considered a body mass index (BMI) $\geq 25 \mathrm{~kg} / \mathrm{m} 2$, and obesity was considered a BMI $\geq 30 \mathrm{~kg} / \mathrm{m} 2$. Hypertension was defined based on systolic blood pressure $\geq 140$, diastole $\geq 90$,or taking antihypertensive medications(25) and was measured using a mercury barometer (26)Fasting blood glucose was measured after ten hours using the standard enzyme method 
(26)Diabetes was defined based on blood sugar $\geq 126 \mathrm{mg} / \mathrm{dL}$ or the use of antidiabetic drugs (24). The lipid profile was measured using a photometric method with a calorimetry enzyme device (CHODPAP) (24). Hypertriglyceridemia was defined as TG levels $\geq 150 \mathrm{mg} / \mathrm{dl}$. Uric acid (UA) was measured by a biochemical autoanalyzer, model BT 3000 (Italy), and Man kits (Tehran, Iran) (UA).

Hyperuricemia was defined as the threshold cut-off point of the 75th percentile of hyperuricemia, which was equal to $5.6 \mathrm{mg} / \mathrm{dl}$ for men and $4.6 \mathrm{mg} / \mathrm{dl}$ for women. Smoking was classified according to selfreported current smoker and consuming at least 10 cigarettes in the last three months. A family history of CVD was considered according to self-reports of having a history of CVD in the first relative family as less than 55 years old in the brother or father or less than 65 years old in the sister or mother. Physical activity was measured according to the International Physical Activity Questionnaires (IPAQ) and was divided into two categories (yes/no) based on physical activity $<150$ minutes per week. The level of education was divided into elementary, high school, diploma and university degree based on the individuals' selfreports.

\subsection{Eligibility Criteria}

The inclusion criteria for the study were residence in Yazd for at least 1 year, age in the range of 2074 years, and being free from confirmed cardiovascular disease at baseline. Additionally, those who had BMI $\leq 24.9$, were not available or their cardiovascular disease information was not available during follow-up were excluded from this study.

\subsection{Outcome Definition}

In this study, 866 participants suffering from obesity and overweight and free from CVD disease at baseline were investigated. CAD was defined as a history of CABG, PTCA, and coronary artery stenosis on angiography and positive Rose Angina Questionnaire and ischemic findings on electrocardiography (ECG). The competing risk was considered all deaths from noncardiac causes (all deaths from cancer, accident, CVA, unknown, retinopathy, infection, aging).

\subsection{Statistical Analyses}

Basic characteristics of patients according to coronary artery disease status were reported as the mean \pm standard deviation for quantitative variables and as frequency (\%) for qualitative variables. The mean survival and 95\% confidence interval were estimated using the Kaplan-Meier method. The model of competing risks based on cause-specific hazard and subdistribution (Fine-Gray) functions was used to determine the risk factors for coronary artery disease in overweight and obese individuals. The significance level of the test was considered $20 \%$ in the univariate model and $5 \%$ in the multivariate model. Proportional hazards were assumed in both models. The cumulative incidence of coronary artery disease was calculated using the following distribution function model.

\subsubsection{Kaplan-Meier}


Kaplan and Meier first published an article in 1985 on how to deal with censored observations(27). The Kaplan-Meier method is a nonparametric estimator of maximum likelihood(28), which calculates the probability of survival of individuals up to a certain time after a particular treatment at short intervals (despite censorship of observations)(29). This probability is calculated by multiplying the survival ratio at the desired interval by the survival ratio up to the previous interval(30).

Suppose that $t_{1}, t_{2}, \ldots, t_{k}$ indicates the time of the events in chronological order, $e_{i}$ is the number of events at time $t_{i}$, and $r_{i}$ is the number of censored times or times of events $\geq t_{i}$. (28).

$$
S_{K M(t)=\prod i t i<t \frac{r_{i}-\varepsilon_{i}}{e_{i}}}
$$

\subsubsection{Cause-Specific Cox Hazard Model}

The model is based on the cause-specific hazard function defined as:

$$
\gamma_{k}^{c s}(t)=\lim _{\Delta t \rightarrow 0} \frac{\operatorname{Prob}\{t \leq T<t+\Delta t, D=k \mid T \geq t\}}{\Delta t}
$$

T denotes the time from a well-defined time origin until an event occurs. The cause-specific hazard function measures the instantaneous rate for occurrence of the $k^{\text {th }}$ event in individuals who are still at the risk of the event.

\subsubsection{Fine-Gray Model}

The cumulative distribution function model was first proposed by Fine-Gray(31) and examines the relationship between Curtis and cumulative incidence based on subdistribution hazards $(15,32)$.

experienced the event(32). The set at risk includes both individuals who have not experienced the desired event prior to time t or have experienced a competing event before this time $(11,15)$

The risk set includes both individuals who have not experienced the event prior to time $t$ or who have experienced a competing event prior to this time $(11,15)$.

$$
\gamma_{k}^{c d}(t)=\lim _{\Delta t \rightarrow 0} \frac{\operatorname{Prob}\{t \leq T<t+\Delta t, D=k \mid T>t \cup(T<t \cap K \neq k)\}}{\Delta t}
$$

\subsubsection{Cumulative Incidence Function}

This function denotes the probability of failing from the $k^{\text {th }}$ event before giving time and before experiencing various types of events.

$$
C I F_{k}(t)=\operatorname{Pr}(T=, D=k)(2)
$$

D denotes the type of the event that happens (11). 


\subsubsection{Ethics approval and consent to participate}

The present study was ethically approved by the Shahid sadoughi University of Medical Sciences' ethics committee (ethics code: IR.SSU.SPH.REC.1397.062). Informed consents were obtained from study participants at the initial and the follow-up phase. All provisions of the Declaration of Helsinki adhered in our study, especially appropriate ethical and scientific review.

\section{Result}

This study consisted of 866 participants with a mean age of $48 \pm 13.6$ and BMI 29.84 \pm 5.43 . The median duration of follow-up was 9 years; $92(10.6 \%)$ new cases of CAD and 34 (4\%) cases of noncardiac deaths occurred. The baseline characteristics according to the patients' outcomes are shown in Table 1. Participants with CAD were older and more likely male and diabetic and had no physical activity or primary education. Table 1. The baseline characteristics in CAD and non-CAD patients. 
Table 1

The baseline characteristics in CAD and Non-CAD patients

\begin{tabular}{|c|c|c|c|c|}
\hline & CAD & Non-CAD & Non-cardia death & Total \\
\hline \multirow[t]{2}{*}{ variables } & Mean \pm SD & Mean \pm SD & Mean \pm SD & Mean \pm SD \\
\hline & Frequency (\%) & Frequency (\%) & Frequency (\%) & Frequency (\%Total) \\
\hline Age & $58.04 \pm 9.94$ & $47.85 \pm 12.95$ & $60.37 \pm 11.17$ & $48.86 \pm 13.64$ \\
\hline BMI & $30.16 \pm 16.57$ & $29.55 \pm 7.085$ & $32.036 \pm 20.39$ & $29.84 \pm 5.43$ \\
\hline SBP & $138.4 \pm 17.52$ & $128.63 \pm 14.06$ & $134.86 \pm 13.34$ & $129.93 \pm 16.99$ \\
\hline DBP & $86.22 \pm 9.26$ & $83.39 \pm 8.5$ & $83.69 \pm 7.97$ & $83.58 \pm 8.79$ \\
\hline TG & $211.61 \pm 88.53$ & $187.43 \pm 111.55$ & $191.89 \pm 70.96$ & $189.73 \pm 109.96$ \\
\hline LDL & $121.14 \pm 31.98$ & $110.49 \pm 36.93$ & $117.83 \pm 33.7$ & $111.50 \pm 35.92$ \\
\hline HDL & $53.93 \pm 13.51$ & $54.22 \pm 13.31$ & $52.71 \pm 13.31$ & $53.81 \pm 13.1$ \\
\hline UA & $4.87 \pm 1.45$ & $4.48 \pm 1.25$ & $4.48 \pm 1.28$ & $4.51 \pm 1.26$ \\
\hline \multicolumn{5}{|l|}{ DM } \\
\hline yes & $35(19.9)$ & $125(71)$ & $16(9.1)$ & $176(20.3)$ \\
\hline no & $57(8.3)$ & 614(89) & $19(2.8)$ & $690(79.7)$ \\
\hline \multicolumn{5}{|l|}{ Sex } \\
\hline male & $53(13.8)$ & 313(81.3) & $19(4.9)$ & $385(44.5)$ \\
\hline female & $39(8.1)$ & 426(88.6) & $16(3.3)$ & $481(55.5)$ \\
\hline \multicolumn{5}{|c|}{ Current Smoking } \\
\hline yes & $12(15)$ & $60(75)$ & $8(10)$ & $80(9.7)$ \\
\hline no & $75(10.1)$ & $646(86.8)$ & 23(3.1) & $744(90.3)$ \\
\hline \multicolumn{5}{|c|}{ Family history } \\
\hline yes & $9(5.7)$ & 143(90.5) & $6(3.8)$ & $158(18.2)$ \\
\hline no & $83(11.7)$ & $596(84.2)$ & $29(4.1)$ & $708(81.8)$ \\
\hline \multicolumn{5}{|c|}{ Physical activity } \\
\hline yes & $14(8)$ & 155(89.1) & $5(2.9)$ & 174(30.7) \\
\hline no & $56(14.2)$ & 319(81.2) & $18(4.6)$ & $393(69.3)$ \\
\hline
\end{tabular}




\begin{tabular}{|lllll|}
\hline & CAD & Non-CAD & \multicolumn{1}{l|}{ Non-cardia death } & Total \\
\hline Primary & $74(14.1)$ & $428(80.8)$ & $27(5.1)$ & $525(61.4)$ \\
\hline High school & $17(6.6)$ & $239(92.3)$ & $3(2.1)$ & $259(30.3)$ \\
\hline Academic & $1(1.4)$ & $65(91.5)$ & $5(7)$ & $71(8.3)$ \\
\hline $\begin{array}{l}\text { BMl: Body mass index, SBP: Systolic blood pressure, DBP: } \text { diastolic blood pressure, DM: Diabetes } \\
\text { mellitus, TG: Triglycerides, LDL: low density lipoproteins, HDL: high density lipoprotein, UA: Uric Acid }\end{array}$ \\
\hline
\end{tabular}

The cumulative incidence of any CAD at the 5-year and 10-year follow-ups was approximately $6.8 \%$ and $10.6 \%$, respectively, and approximately $4.6 \%$ and $8.5 \%$, respectively, for all other noncardiac deaths (Figure 2).

\subsection{Hazard Models for Coronary Artery Disease}

The estimated unadjusted hazard ratios and their confidence interval estimates for univariate analysis are shown in Table 2. The results found in both models with no confounding variables of age, sex (male), SPB, DM, hypertriglyceridemia, LDL, uric acid, current smoking, education level (inverse) and physical activity (inverse) were significantly associated with the risk of CAD (Table 2). The proportional hazards assumption was checked for any covariate and was verified by the lack of significant interactions with the survival time. 
Table 2

Univariate competing risk model for incidence of CAD in presence non-cardiac death as competing event

\begin{tabular}{|c|c|c|c|c|}
\hline Baseline Characteristics & $\mathrm{HR}(95 \% \mathrm{Cl})$ & P-value & SHR (95\% Cl) & P-value \\
\hline Age & $1.06(1.0411 .079)$ & $<0.001^{*}$ & $1.06(1.041 .07)$ & $<0.001^{*}$ \\
\hline SBP & 1.035 (1.0231.047) & $<0.001^{\star}$ & $1.03(1.021 .05)$ & $<0.001^{*}$ \\
\hline DBP & $1.03(1.0101 .053)$ & $0.004^{*}$ & $1.03(1.011 .05)$ & $0.002^{\star}$ \\
\hline Diabetes Mellitus & $2.57(1.6903 .922)$ & $<0.001^{\star}$ & $2.52(1.673 .82)$ & $<0.001^{*}$ \\
\hline HDL & $0.99(0.9841 .105)$ & 0.94 & $0.998(0.991)$ & 0.5 \\
\hline LDL & $1.007(1.0011 .012)$ & $0.018^{*}$ & $1.01(11.01)$ & $0.004^{\star}$ \\
\hline Acid Uric & $1.24(1.0681 .442)$ & $0.005^{*}$ & $1.24(1.061 .45)$ & $0.006^{\star}$ \\
\hline Hypertriglyceridemia(yes) & 2.188(1.3743.484) & $0.001^{*}$ & $2.15(1.353 .42)$ & $0.001^{*}$ \\
\hline Sex (female) & $0.560(0.3710 .847)$ & $0.006^{*}$ & $0.568(0.3770 .856)$ & $0.006^{\star}$ \\
\hline Current Smoking (yes) & $1.53(0.8432 .853)$ & $0.16^{*}$ & $1.55(0.8422 .85)$ & $0.16^{\star}$ \\
\hline Physical activity(yes) & $0.533(0.2970 .957)$ & $0.035^{*}$ & $0.549(0.3070 .982)$ & $0.042^{\star}$ \\
\hline Education level & $0.401(0.2540 .634)$ & $<0.001^{*}$ & & \\
\hline low & Ref & & & \\
\hline moderate & $0.442(0.2610 .749)$ & $0.002^{\star}$ & $0.447(0.2660 .754)$ & $0.0025^{\star}$ \\
\hline high & $0.097(0.0140 .70)$ & 0.021 & $0.0949(0.0130 .691)$ & $0.02^{\star}$ \\
\hline \multicolumn{5}{|c|}{$\begin{array}{l}{ }^{*} \mathrm{P}<0.2 \text {, HR: hazard ratio, SHR: sub-distribution hazard ratio, Cl: confidence interval, CAD: Coronary } \\
\text { Artery Disease, BMI: Body mass index, SBP: Systolic blood pressure, DBP: diastolic blood pressure, } \\
\text { DM: Diabetes mellitus, TG: Triglycerides, LDL: low density lipoproteins, HDL: high density lipoprotein, } \\
\text { UA: Uric Acid }\end{array}$} \\
\hline
\end{tabular}

It is important to remember that the 2 models have different interpretations when interpreting the regression coefficients from the cause-specific hazard models and the subdistribution hazard models. 
Table 3

Multivariate competing risk model for incidence of CAD in presence non-cardiac death as competing event

\begin{tabular}{|llcll|}
\hline Baseline characteristics & HR $(95 \%$ Cl) & P-value & SHR (95\% Cl) & P-value \\
\hline Age & $1.034(1.011 .058)$ & ${ }^{*} 0.005$ & $1.032(1.021 .054)$ & $0.003^{*}$ \\
\hline SBP & $1.021(1.0061 .037)$ & ${ }^{*} 0.007$ & $1.026(1.00391 .049)$ & $0.02^{*}$ \\
\hline DM (yes) & $1.92(1.023 .089)$ & ${ }^{*} 0.007$ & $1.966(1.2743 .03)$ & $0.002^{*}$ \\
\hline Sex(female) & $0.566(0.3570 .897)$ & ${ }^{*} 0.015$ & $0.587(0.3580 .961)$ & $0.034^{*}$ \\
\hline Hypertriglyceridemia (yes) & $1.904(1.1633 .115)$ & ${ }^{*} 0.01$ & $1.858(1.1373 .05)$ & $0.013^{*}$ \\
\hline UA & $1.21(1.0131 .447)$ & ${ }^{*} 0.036$ & $1.21(1.0131 .464)$ & $0.03^{*}$ \\
\hline Education level & & & & \\
\hline low & Ref & & Ref & \\
\hline $\begin{array}{l}\text { moderate } \\
\text { high }\end{array}$ & $0.772(0.421 .420)$ & 0.405 & $0.735(0.4021 .344)$ & 0.32 \\
\hline $\begin{array}{l}* \text { P<0.05, HR: hazard ratio, SHR: sub-distribution hazard ratio, Cl: confidence interval, CAD: Coronary } \\
\text { Artery Disease, SBP: Systolic blood pressure, DM: Diabetes mellitus, TG: Triglycerides, UA: Uric Acid }\end{array}$ \\
\hline
\end{tabular}

The multivariate cause-specific hazard model showed that variables DM (HR: 1.92, $P<0.01$ ), hypertriglyceridemia (HR: 1.904, $P<0.05)$, uric acid (HR: 1.26, $P<0.01)$, age (HR: 1.034, $P<0,01)$, SBP (HR: $1.021, P<0.01)$, associated with an increased hazard rate of CAD and university level of education (HR: $0.125, P<0.05)$ and female sex (HR: $0.566, P<0.01)$ were associated with a reduced hazard rate of $C A D$. Similarly, in the multivariate fine-gray models, DM (SHR: 1.966, P<0.01), hypertriglyceridemia (SHR: 1.858, $P<0.05)$, uric acid (SHR: 1.21, $P<0.05$ ), age (SHR: 1.032, $P<0.01)$, SBP (SHR: 1.026, $P<0.05)$, university level of education (SHR: 0.105, $\mathrm{P}<0.05)$, and sex (SHR: 0.587, $\mathrm{P}<0.05)$ were associated with an increased risk of CAD (Table 3).

\section{Discussion}

This study focused on the use of cause-specific hazard regression and Fine-Gray models versus competing risk as death due to noncardiac causes to investigate the effect of risk factors related to the incidence of coronary artery disease in overweight and obese populations of the city of Yazd, aged 2074 years, according to cohort data. In the present study, the 10-year cumulative crude incidence of coronary artery disease was $10.6 \%$ vs $6.8 \%$. 
The percentage of the competing cause as noncardiac deaths. Additionally, in line with previous studies, older age(33), higher systolic blood pressure(33), higher uric acid, male sex, diabetes(33), and hypertriglyceridemia increased the likelihood of developing coronary artery disease, and those with higher education (inversely) were less likely to develop CAD.

In the present study, the cause-specific and Fine-Gray models performed similarly in the 10-year prediction of coronary artery disease, in line with the study of Wilber et al.(34). According to the findings, the causespecific hazard and subdistribution hazard of coronary artery disease associated with a per ten-year increase in age, systolic blood pressure, and uric acid had a very slight difference and similarly the causespecific hazard and subdistribution hazard of coronary artery disease associated in during ten-year in diabetics, hypertriglyceridemia and higher education were similar.

However, Wilber's reported that $18 \%$ and $8 \%$ of people were classified at high risk of coronary artery disease according to the Cox and Fine-Gray models, respectively(34). Puddu et al. also showed that the use of the CIF model based on the Fine-Gray model was more appropriate and could avoid overestimations of the Kaplan-Meier method based on methods such as the Cox model(35). The study of Mackenzie reported that Cox proportional hazard models were not a good predictor of individual risk (36).

According to our findings, a per ten-year increase in age was associated with a $3.3 \%$ increase in the cause-specific hazard rate of coronary artery disease and a similar 3.2\% increase in the risk of coronary artery disease (relative incidence) for at-risk individuals (Table 3). Age is an irreversible risk factor that plays a vital role in mortalities due to cardiovascular disease, remaining as a predictor variable in multivariate models even after adjusting for other confounders(37-39). In the present study, most cases of coronary artery disease occurred in the age of 65-74 years old.

Sex is an important risk factor for CVD in aging adults (37). In our study, men developed 52 (13.5\%), and women developed $39(7.9 \%)$ new cases of CAD. Consistent with the results of this study, Tsiampalis et al. reported that women were less likely to die from CHD, but Govender et al. reported that women were 1.96 times more likely than men to have a recurrence of cardiovascular disease(40).

Our findings showed that a per10-year increase in systolic blood pressure was associated with a $25.4 \%$ increase in the cause-specific hazard rate of coronary artery disease and, similarly, a $26 \%$ increase in the relative incidence of at-risk coronary artery disease. Consistent with our results, Puddu et al., using the Cox model (HR: 1.402, 95\% Cl: 1.3931.410, P <0.001) and Fine-Gray (SHR: 1.152, 95\% Cl: 1.1451.159, P $<0.001$ ), showed that increased systolic blood pressure was associated with a higher risk of CHD death(35). Ramezanian et al. also showed in the multivariate Cox model that a one-unit increase in systolic and diastolic blood pressure was associated with a $1 \%$ increase in the risk of CAD. Using a competing risk model, Dianatkhah et al. reported that hypertension was associated with a higher risk of cardiovascular disease(33). 
In this study, diabetic patients were more likely to develop CAD. The cause-specific hazard and subdistribution hazard of coronary artery disease in high-risk diabetic patients were $2.5 \%$ and $2.5 \%$ higher than those in nondiabetic patients, respectively, and $19.9 \%$ of diabetic patients at the beginning of the study ( $n=176)$ developed CAD over ten years. Diabetic patients have a worse prognosis of CAD, so the risk of cardiac death is 2 to 4 times higher in these patients than in others(41). The increase in obesity epidemic in the world has led to the prevalence of CAD and diabetes(42). In many countries, the prevalence of diabetes in patients with coronary artery disease is reported to be $50 \%$ (43). Ramazanian et al. showed in the multivariate Cox model that each unit increase in fasting blood sugar increased the risk of CAD to $10 \%$ and $13 \%$ (44) in women and men, respectively. Tsiampalis et al. showed that increasing each unit in fasting blood sugar increased the risk of $\mathrm{CHS}$ death by $0.3 \%$, and women were less likely to die from $\mathrm{CHD}(45)$. The cause-specific hazard and subdistribution hazard of CAD were approximately twice as high in at-risk people with hypertriglyceridemia as those with normal triglycerides. Additionally, a per 10-year increase in uric acid was associated with a $21 \%$ increase in the cause-specific hazard rate and subdistribution hazard of CAD in at-risk individuals. Longitudinal epidemiological studies have shown that serum uric acid is a predictor for the incidence of cardiovascular diseases and cardiac deaths(46). A study showed an association between elevated uric acid levels and overweight and obesity(47).

Additionally, our findings showed that the risk of coronary artery disease decreased with increasing level of education, taking into account the competing event (death due to noncardiac causes) (Table 3).

Education is one of the socioeconomic factors affecting the risk of cardiovascular diseases and is completed by adulthood $(48,49)$. In a cohort study on 9226 patients without a history of cardiovascular disease, Dégano et al. reported that the risk of cardiovascular disease was lower in people with a university degree than in those with a lower or primary education ( $\mathrm{HR}=0.51,95 \% \mathrm{Cl}: 0.300 .85)(49)$. It seems that preventive strategies should be planned and made available to individuals according to their level of education(49). For example, some people may have learning disabilities, increasing lifestylerelated risk for CVD in these individuals(48). It also seems that people with higher education will have a healthier lifestyle despite economic changes, and the risk of cardiovascular disease will be reduced in these people(48). The findings of this study are consistent with other studies.

The at-risk group in the Kaplan-Meier model does not include individuals who experience a competing event before the event in question(18). The Cox model considered competing risk as censorship when examining the effect of variables on a particular cause(18). One of the challenges of competing risks in medical research on survival analysis is the one-to-one mismatch between the hazard function (rate) and the cumulative incidence function(18). In the Cox model, there is a one-to-one correspondence between the hazard function and the cumulative incidence function; therefore, the hazard ratio is equivalent to the rate in epidemiology and reflects the risk in population studies(18). The cumulative incidence function in the cause-specific model is affected by both the cause-specific function of the desired event and the competing event(34). The results of the cause-specific model for a particular variable should not be reported as an increase or decrease in the incidence of the disease because the effect of the variables on both the cause-specific hazard function and the competing event must be determined to examine the effect of variables on the incidence of the disease $(34,50)$. 
The subdistribution function still keeps those who experienced the competing risk before the desired event in the at-risk set $(11,50)$. For example, if the competing risk is death due to cardiac causes, deceased heart patients are no longer at risk for the disease; therefore, the subdistribution function is not considered a standard epidemiological rate as a hazard ratio $(51,52)$. The hazard ratio in the Fine-Gray model is interpreted as a relative change in the desired event rate in people who have not experienced the desired event and the competing cause by a certain time(50). and directly determines the effect of variables on disease incidence $(14,18)$. In general, it is suggested to use the cause-specific hazard function for studies of disease etiology $(11,53)$. and the subdistributed hazard function for prognostic, predictive, and risk score classification in survival studies $(11,14,54)$.

\section{Conclusion}

The two cause-specific and Fine-Gray models performed similarly in a per 10-year prediction of coronary artery disease in the present study. The findings showed that the hazard ratio and confidence interval for all significant variables in the incidence of coronary artery disease were similar in the final models of cause-specific and subdistribution and acted similarly in terms of the significant variable. The findings showed that in the presence of competing risks, age, systolic blood pressure, male sex, uric acid, history of diabetes, history of hypertriglyceridemia and high level of education (inversely) were associated with increased risk of coronary artery disease in overweight and obese individuals. Considering the overestimation, the Cox model censors the competing risks and overestimates the effect of covariates on the risk of disease. Therefore, the use of competing risk models in the presence of a competing cause is emphasized when interpreting survival studies. However, it should be noted that the relationship between variables and the cause-specific hazard function is not similar to their dependence on the cumulative incidence function; thus, the risk of variables should not be interpreted similarly in these two models.

\section{Declarations}

\section{Acknowledgements}

We appreciate Dr Seyed Mahmood Sadr-Bafghi and Dr MohammadHossein Soltani for their cooperation in design and conduct of the study. Dr Leila Hadiani for visiting of our patients and collection data. Dr HamidReza Dehghan for visiting of our patients and data bank and data management and review of our article. MS. Maryam Askari, Mr. Ahmad Karimi and Mr. Mojtaba MohammadHosseini for for Data extraction. We especially thank the personnel of Yazd Heart Research Center, headed by S Reyhani, R Atashkoh, S Lesan, and R Abedini who had full cooperation in this study.

\section{Author contributions}

F.M. performed the data analysis and drafted the manuscript. M.A. developed the analysis plan and critically reviewed the manuscript. M.N. reviewed critical manuscript and access to data and responsibility for the manuscript contents. All authors reviewed the manuscript 


\section{Corresponding author:}

Correspondence to Fatemeh Majidpour

\section{Ethics approval and consent to participate}

The present study was ethically approved by the Shahid sadoughi University of Medical Sciences' ethics committee (ethics code: IR.SSU.SPH.REC.1397.062). Informed consents were obtained from study participants at the initial and the follow-up phase. All provisions of the Declaration of Helsinki adhered in our study, especially appropriate ethical and scientific review.

\section{Consent for publication}

Not applicable.

\section{Competing Interests}

The authors declare that they have no competing interests.

\section{Funding}

All parts of this research work, design of the study, data collection, analysis, interpretation of data, and writing of the manuscript were funded by the Research, Department Biostatics \& Epidemiology, Health Faculty, Shahid Sadoughi University of Medical Sciences, Yazd, Iran. The funding body played no role in publication costs.

\section{Availability of data and material}

The datasets used and analysed during the current study are available from Seyedeh Mahdieh Namayandeh author on reasonable request.

\section{References}

1. Ralapanawa U, Sivakanesan R. Epidemiology and the Magnitude of Coronary Artery Disease and Acute Coronary Syndrome: A Narrative Review. Journal of Epidemiology and Global Health. 2021.

2. Malakar AK, Choudhury D, Halder B, Paul P, Uddin A, Chakraborty S. A review on coronary artery disease, its risk factors, and therapeutics. Journal of cellular physiology. 2019;234(10):16812-23.

3. Roth GA, Mensah GA, Johnson CO, Addolorato G, Ammirati E, Baddour LM, et al. Global burden of cardiovascular diseases and risk factors, 1990-2019: update from the GBD 2019 study. Journal of the American College of Cardiology. 2020;76(25):2982-3021.

4. Sarrafzadegan N, Mohammmadifard N. Cardiovascular disease in Iran in the last 40 years:

prevalence, mortality, morbidity, challenges and strategies for cardiovascular prevention. Archives of Iranian medicine. 2019;22(4):204-10. 
5. Cercato C, Fonseca F. Cardiovascular risk and obesity. Diabetology \& metabolic syndrome. 2019;11(1):1-15.

6. Ortega FB, Lavie CJ, Blair SN. Obesity and cardiovascular disease. Circulation research. 2016;118(11):1752-70.

7. Powell-Wiley TM, Poirier P, Burke LE, Després J-P, Gordon-Larsen P, Lavie CJ, et al. Obesity and cardiovascular disease: a scientific statement from the American Heart Association. Circulation. 2021;143(21):e984-e1010.

8. Collaboration APCS. Body mass index and cardiovascular disease in the Asia-Pacific Region: an overview of 33 cohorts involving 310000 participants. International journal of epidemiology. 2004;33(4):751-8.

9. Namayandeh SM, Sadr S, Ansari Z, Rafiei M. A cross-sectional study of the prevalence of coronary artery disease traditional risk factors in Yazd urban population, Yazd healthy heart project. 2011.

10. Wolbers M, Koller MT, Stel VS, Schaer B, Jager KJ, Leffondre K, et al. Competing risks analyses: objectives and approaches. European heart journal. 2014;35(42):2936-41.

11. Austin PC, Lee DS, Fine JP. Introduction to the analysis of survival data in the presence of competing risks. Circulation. 2016;133(6):601-9.

12. Huebner M, Wolkewitz M, Enriquez-Sarano M, Schumacher M. Competing risks need to be considered in survival analysis models for cardiovascular outcomes. The Journal of thoracic and cardiovascular surgery. 2017;153(6):1427-31.

13. Haushona N, Esterhuizen TM, Thabane L, Machekano R. An empirical comparison of time-to-event models to analyse a composite outcome in the presence of death as a competing risk. Contemporary Clinical Trials Communications. 2020;19:100639.

14. Abdel-Qadir H, Fang J, Lee DS, Tu JV, Amir E, Austin PC, et al. Importance of considering competing risks in time-to-event analyses: application to stroke risk in a retrospective cohort study of elderly patients with atrial fibrillation. Circulation: Cardiovascular Quality and Outcomes. 2018;11(7):e004580.

15. Jeon Y, Lee WK. Competing Risk Model in Survival Analysis. Cardiovascular Prevention and Pharmacotherapy. 2020;2(3):77-84.

16. Puddu PE, Amaduzzi PL, Ricci B. Coronary heart disease incidence and competing risks: an important issue. Journal of geriatric cardiology: JGC. 2017;14(7):425.

17. Buzkova P. Competing risk of mortality in association studies of non-fatal events. Plos one. 2021;16(8):e0255313.

18. Zhang Z. Survival analysis in the presence of competing risks. Annals of translational medicine. 2017;5(3).

19. Talaei M, Sarrafzadegan N, Sadeghi M, Oveisgharan S, Marshall T, Thomas GN, et al. Incidence of cardiovascular diseases in an Iranian population: the Isfahan Cohort Study. Archives of Iranian medicine. 2013;16(3):0-. 
20. Oh WS, Yoon S, Noh J, Sohn J, Kim C, Heo J. Geographical variations and influential factors in prevalence of cardiometabolic diseases in South Korea. PloS one. 2018;13(10):e0205005.

21. Trias-Llimós S, Pennells L, Tverdal A, Kudryavtsev AV, Malyutina S, Hopstock LA, et al. Quantifying the contribution of established risk factors to cardiovascular mortality differences between Russia and Norway. Scientific reports. 2020;10(1):1-8.

22. Sayers A, Evans JT, Whitehouse MR, Blom AW. Are competing risks models appropriate to describe implant failure? Acta orthopaedica. 2018;89(3):256-8.

23. Teixeira L, Rodrigues A, Carvalho MJ, Cabrita A, Mendonça D. Modelling competing risks in nephrology research: an example in peritoneal dialysis. BMC nephrology. 2013;14(1):1-8.

24. Namayandeh SM, Karimi A, Fallahzadeh H, Rahmanian M, Bafghi SMS, Soltani M, et al. The incidence rate of diabetes mellitus (type II) and its related risk factors: A 10-year longitudinal study of Yazd Healthy Heart Cohort (YHHC), Iran. Diabetes \& Metabolic Syndrome: Clinical Research \& Reviews. 2019;13(2):1437-41.

25. Whelton PK, Carey RM, Aronow WS, Casey DE, Collins KJ, Dennison Himmelfarb C, et al. 2017 ACC/AHA/AAPA/ABC/ACPM/AGS/APhA/ASH/ASPC/NMA/PCNA guideline for the prevention, detection, evaluation, and management of high blood pressure in adults: a report of the American College of Cardiology/American Heart Association Task Force on Clinical Practice Guidelines. Journal of the American College of Cardiology. 2018;71(19):e127-e248.

26. Mohammadhoseini M, Namayandeh SM, Fallahzadeh H, Majidpour F, Sadr-Bafghi SM, Soltani M, et al. Population Attributable Risk of Hyperuricemia in Hypertension Incidence in 20-74-Year-Old Population during a 10-Year Longitudinal Cohort: Yazd Healthy Heart Cohort. Iranian Journal of Public Health. 2020;49(10):1977.

27. Rich JT, Neely JG, Paniello RC, Voelker CC, Nussenbaum B, Wang EW. A practical guide to understanding Kaplan-Meier curves. Otolaryngology-Head and Neck Surgery. 2010;143(3):331-6.

28. Eleuteri A, Tagliaferri R, Milano L, de Laurentiis M. Information Geometry for Survival Analysis and Feature Selection by Neural Networks. Outcome Prediction in Cancer: Elsevier; 2007. p. 171-89.

29. Goel MK, Khanna P, Kishore J. Understanding survival analysis: Kaplan-Meier estimate. International journal of Ayurveda research. 2010;1(4):274.

30. Damato B, Taktak A. Survival after treatment of intraocular melanoma. Outcome Prediction in Cancer: Elsevier; 2007. p. 27-41.

31. Fine JP, Gray RJ. A proportional hazards model for the subdistribution of a competing risk. Journal of the American statistical association. 1999;94(446):496-509.

32. Alipour A, Shokri A, Yasari F, Khodakarim S. Introduction to Competing Risk Model in the Epidemiological Research. International Journal of Epidemiologic Research. 2018;5(3):98-102.

33. Dianatkhah $M$, Rahgozar M, Talaei M, Karimloua M, Sadeghi M, Oveisgharan $S$, et al. Comparison of competing risks models based on cumulative incidence function in analyzing time to cardiovascular diseases. ARYA atherosclerosis. 2014;10(1):6. 
34. Wolbers M, Koller MT, Witteman JC, Steyerberg EW. Prognostic models with competing risks: methods and application to coronary risk prediction. Epidemiology. 2009:555-61.

35. Puddu PE, Piras P, Menotti A. Lifetime competing risks between coronary heart disease mortality and other causes of death during 50 years of follow-up. International journal of cardiology. 2017;228:35963.

36. MacKenzie G, Greig M, Hay I, Pemberton J. Competing risk analysis of factors related to long-term incidence of CHD. J Epidemiol Community Health. 2017;71(1):33-6.

37. Rodgers JL, Jones J, Bolleddu SI, Vanthenapalli S, Rodgers LE, Shah K, et al. Cardiovascular risks associated with gender and aging. Journal of cardiovascular development and disease. 2019;6(2):19.

38. Costa E, Santos-Silva A, Paúl C, González Gallego J. Aging and cardiovascular risk. Hindawi; 2015.

39. Dhingra R, Vasan RS. Age as a risk factor. Medical Clinics. 2012;96(1):87-91.

40. Govender RD, Al-Shamsi S, Soteriades ES, Regmi D. Incidence and risk factors for recurrent cardiovascular disease in middle-eastern adults: a retrospective study. BMC cardiovascular disorders. 2019;19(1):1-7.

41. Aronson D, Edelman ER. Coronary artery disease and diabetes mellitus. Cardiology clinics. 2014;32(3):439-55.

42. Al-Nozha MM, Ismail HM, Al Nozha OM. Coronary artery disease and diabetes mellitus. Journal of Taibah University Medical Sciences. 2016;11(4):330-8.

43. AlHabib KF, Sulaiman K, Al-Motarreb A, Almahmeed W, Asaad N, Amin H, et al. Baseline characteristics, management practices, and long-term outcomes of Middle Eastern patients in the Second Gulf Registry of Acute Coronary Events (Gulf RACE-2). Annals of Saudi medicine. 2012;32(1):9-18.

44. Ramezankhani A, Bagherzadeh-Khiabani F, Khalili D, Azizi F, Hadaegh F. A new look at risk patterns related to coronary heart disease incidence using survival tree analysis: 12 Years Longitudinal Study. Scientific reports. 2017;7(1):1-11.

45. Tsiampalis T, Panagiotakos D. Competing risks' analysis in cardiovascular disease epidemiology;The case of ATTICA study (2002-2012).

46. Chang C-C, Wu C-H, Liu L-K, Chou R-H, Kuo C-S, Huang P-H, et al. Association between serum uric acid and cardiovascular risk in nonhypertensive and nondiabetic individuals: The Taiwan I-Lan Longitudinal Aging Study. Scientific reports. 2018;8(1):1-6.

47. Duan Y, Liang W, Zhu L, Zhang T, Wang L, Nie Z, et al. Association between serum uric acid levels and obesity among university students (China). Nutricion hospitalaria. 2015;31(6):2407-11.

48. Kubota Y, Heiss G, MacLehose RF, Roetker NS, Folsom AR. Association of educational attainment with lifetime risk of cardiovascular disease: the Atherosclerosis Risk in Communities Study. JAMA internal medicine. 2017;177(8):1165-72. 
49. Dégano IR, Marrugat J, Grau M, Salvador-González B, Ramos R, Zamora A, et al. The association between education and cardiovascular disease incidence is mediated by hypertension, diabetes, and body mass index. Scientific reports. 2017;7(1):1-8.

50. Austin PC, Fine JP. Practical recommendations for reporting $F$ ine-G ray model analyses for competing risk data. Statistics in medicine. 2017;36(27):4391-400.

51. Warnock DG. Competing risks: you only die once. Nephrology Dialysis Transplantation. 2016;31(7):1033-5.

52. Andersen PK, Geskus RB, de Witte T, Putter H. Competing risks in epidemiology: possibilities and pitfalls. International journal of epidemiology. 2012;41(3):861-70.

53. Lau B, Cole SR, Gange SJ. Competing risk regression models for epidemiologic data. American journal of epidemiology. 2009;170(2):244-56.

54. Koller MT, Raatz H, Steyerberg EW, Wolbers M. Competing risks and the clinical community: irrelevance or ignorance? Statistics in medicine. 2012;31(11-12):1089-97.

\section{Tables}


Table 1. The baseline characteristics in CAD and Non-CAD patients

\begin{tabular}{|c|c|c|c|c|}
\hline & CAD & Non-CAD & Non-cardia death & Total \\
\hline \multirow[t]{2}{*}{ variables } & Mean \pm SD & Mean \pm SD & Mean \pm SD & Mean \pm SD \\
\hline & Frequency (\%) & Frequency (\%) & Frequency (\%) & Frequency (\%Total) \\
\hline Age & $58.04 \pm 9.94$ & $47.85 \pm 12.95$ & $60.37 \pm 11.17$ & $48.86 \pm 13.64$ \\
\hline BMI & $30.16 \pm 16.57$ & $29.55 \pm 7.085$ & $32.036 \pm 20.39$ & $29.84 \pm 5.43$ \\
\hline SBP & $138.4 \pm 17.52$ & $128.63 \pm 14.06$ & $134.86 \pm 13.34$ & $129.93 \pm 16.99$ \\
\hline DBP & $86.22 \pm 9.26$ & $83.39 \pm 8.5$ & $83.69 \pm 7.97$ & $83.58 \pm 8.79$ \\
\hline TG & $211.61 \pm 88.53$ & $187.43 \pm 111.55$ & $191.89 \pm 70.96$ & $189.73 \pm 109.96$ \\
\hline LDL & $121.14 \pm 31.98$ & $110.49 \pm 36.93$ & $117.83 \pm 33.7$ & $111.50 \pm 35.92$ \\
\hline $\mathrm{HDL}$ & $53.93 \pm 13.51$ & $54.22 \pm 13.31$ & $52.71 \pm 13.31$ & $53.81 \pm 13.1$ \\
\hline UA & $4.87 \pm 1.45$ & $4.48 \pm 1.25$ & $4.48 \pm 1.28$ & $4.51 \pm 1.26$ \\
\hline \multicolumn{5}{|l|}{ DM } \\
\hline yes & $35(19.9)$ & $125(71)$ & 16(9.1) & $176(20.3)$ \\
\hline no & $57(8.3)$ & $614(89)$ & $19(2.8)$ & $690(79.7)$ \\
\hline \multicolumn{5}{|l|}{ Sex } \\
\hline male & $53(13.8)$ & $313(81.3)$ & $19(4.9)$ & $385(44.5)$ \\
\hline female & $39(8.1)$ & $426(88.6)$ & 16(3.3) & $481(55.5)$ \\
\hline \multicolumn{5}{|c|}{ Current Smoking } \\
\hline yes & $12(15)$ & $60(75)$ & $8(10)$ & $80(9.7)$ \\
\hline no & $75(10.1)$ & $646(86.8)$ & 23(3.1) & $744(90.3)$ \\
\hline \multicolumn{5}{|c|}{ Family history } \\
\hline yes & $9(5.7)$ & $143(90.5)$ & $6(3.8)$ & $158(18.2)$ \\
\hline no & $83(11.7)$ & $596(84.2)$ & $29(4.1)$ & $708(81.8)$ \\
\hline \multicolumn{5}{|c|}{ Physical activity } \\
\hline yes & $14(8)$ & 155(89.1) & $5(2.9)$ & $174(30.7)$ \\
\hline no & $56(14.2)$ & $319(81.2)$ & $18(4.6)$ & $393(69.3)$ \\
\hline \multicolumn{5}{|c|}{ Education level } \\
\hline Primary & $74(14.1)$ & $428(80.8)$ & $27(5.1)$ & $525(61.4)$ \\
\hline
\end{tabular}




\begin{tabular}{|lllll} 
High school & $17(6.6)$ & $239(92.3)$ & $3(2.1)$ & $259(30.3)$ \\
Academic & $1(1.4)$ & $65(91.5)$ & $5(7)$ & $71(8.3)$
\end{tabular}

BMI: Body mass index, SBP: Systolic blood pressure, DBP: diastolic blood pressure, DM: Diabetes mellitus, TG: Triglycerides, LDL: low density lipoproteins, HDL: high density lipoprotein, UA: Uric Acid

Table 2. Univariate competing risk model for incidence of CAD in presence non-cardiac death as competing event

\begin{tabular}{|c|c|c|c|c|}
\hline Baseline Characteristics & $\mathrm{HR}(95 \% \mathrm{Cl})$ & P-value & SHR $(95 \% \mathrm{Cl})$ & P-value \\
\hline Age & $1.06(1.0411 .079)$ & $<0.001^{*}$ & $1.06(1.041 .07)$ & $<0.001^{*}$ \\
\hline SBP & 1.035 (1.0231.047) & $<0.001^{*}$ & $1.03(1.021 .05)$ & $<0.001^{*}$ \\
\hline DBP & $1.03(1.0101 .053)$ & $0.004^{*}$ & $1.03(1.011 .05)$ & $0.002^{*}$ \\
\hline Diabetes Mellitus & $2.57(1.6903 .922)$ & $<0.001^{*}$ & $2.52(1.673 .82)$ & $<0.001^{*}$ \\
\hline $\mathrm{HDL}$ & $0.99(0.9841 .105)$ & 0.94 & $0.998(0.991)$ & 0.5 \\
\hline LDL & $1.007(1.0011 .012)$ & $0.018^{\star}$ & $1.01(11.01)$ & $0.004^{\star}$ \\
\hline Acid Uric & $1.24(1.0681 .442)$ & $0.005^{\star}$ & $1.24(1.061 .45)$ & $0.006^{*}$ \\
\hline Hypertriglyceridemia(yes) & $2.188(1.3743 .484)$ & $0.001^{*}$ & $2.15(1.353 .42)$ & $0.001^{*}$ \\
\hline Sex (female) & $0.560(0.3710 .847)$ & $0.006^{*}$ & $0.568(0.3770 .856)$ & $0.006^{*}$ \\
\hline Current Smoking (yes) & $1.53(0.8432 .853)$ & $0.16^{*}$ & $1.55(0.8422 .85)$ & $0.16^{*}$ \\
\hline Physical activity(yes) & $0.533(0.2970 .957)$ & $0.035^{\star}$ & $0.549(0.3070 .982)$ & $0.042^{*}$ \\
\hline Education level & $0.401(0.2540 .634)$ & $<0.001^{*}$ & & \\
\hline low & Ref & & & \\
\hline moderate & $0.442(0.2610 .749)$ & $0.002^{\star}$ & $0.447(0.2660 .754)$ & $0.0025^{\star}$ \\
\hline high & $0.097(0.0140 .70)$ & 0.021 & $0.0949(0.0130 .691)$ & $0.02 *$ \\
\hline
\end{tabular}

${ }^{*} \mathrm{P}<0.2$, HR: hazard ratio, SHR: sub-distribution hazard ratio, Cl: confidence interval, CAD: Coronary Artery Disease, BMI: Body mass index, SBP: Systolic blood pressure, DBP: diastolic blood pressure, DM: Diabetes mellitus, TG: Triglycerides, LDL: low density lipoproteins, HDL: high density lipoprotein, UA: Uric Acid 
Table 3. Multivariate competing risk model for incidence of CAD in presence non-cardiac death as competing event

\begin{tabular}{|lllll|}
\hline Baseline characteristics & HR $(95 \% \mathrm{Cl})$ & P-value & SHR $(95 \% \mathrm{Cl})$ & P-value \\
\hline Age & $1.034(1.011 .058)$ & ${ }^{*} 0.005$ & $1.032(1.021 .054)$ & $0.003^{*}$ \\
\hline SBP & $1.021(1.0061 .037)$ & ${ }^{*} 0.007$ & $1.026(1.00391 .049)$ & $0.02^{*}$ \\
\hline DM (yes) & $1.92(1.023 .089)$ & ${ }^{*} 0.007$ & $1.966(1.2743 .03)$ & $0.002^{*}$ \\
\hline Sex(female) & $0.566(0.3570 .897)$ & ${ }^{*} 0.015$ & $0.587(0.3580 .961)$ & $0.034^{*}$ \\
\hline Hypertriglyceridemia (yes) & $1.904(1.1633 .115)$ & ${ }^{*} 0.01$ & $1.858(1.1373 .05)$ & $0.013^{*}$ \\
\hline UA & $1.21(1.0131 .447)$ & ${ }^{*} 0.036$ & $1.21(1.0131 .464)$ & $0.03^{*}$ \\
\hline Education level & & & & \\
\hline low & Ref & & Ref & 0.32 \\
\hline moderate & $0.772(0.421 .420)$ & 0.405 & $0.735(0.4021 .344)$ & $0.027^{*}$ \\
\hline $\begin{array}{l}\text { high } \\
\text { *P<0.05, HR: hazard ratio, SHR: sub-distribution hazard ratio, Cl: confidence interval, CAD: Coronary }\end{array}$ \\
\hline Artery Disease, SBP: Systolic blood pressure, DM: Diabetes mellitus, TG: Triglycerides, UA: Uric Acid \\
\hline
\end{tabular}

\section{Figures}




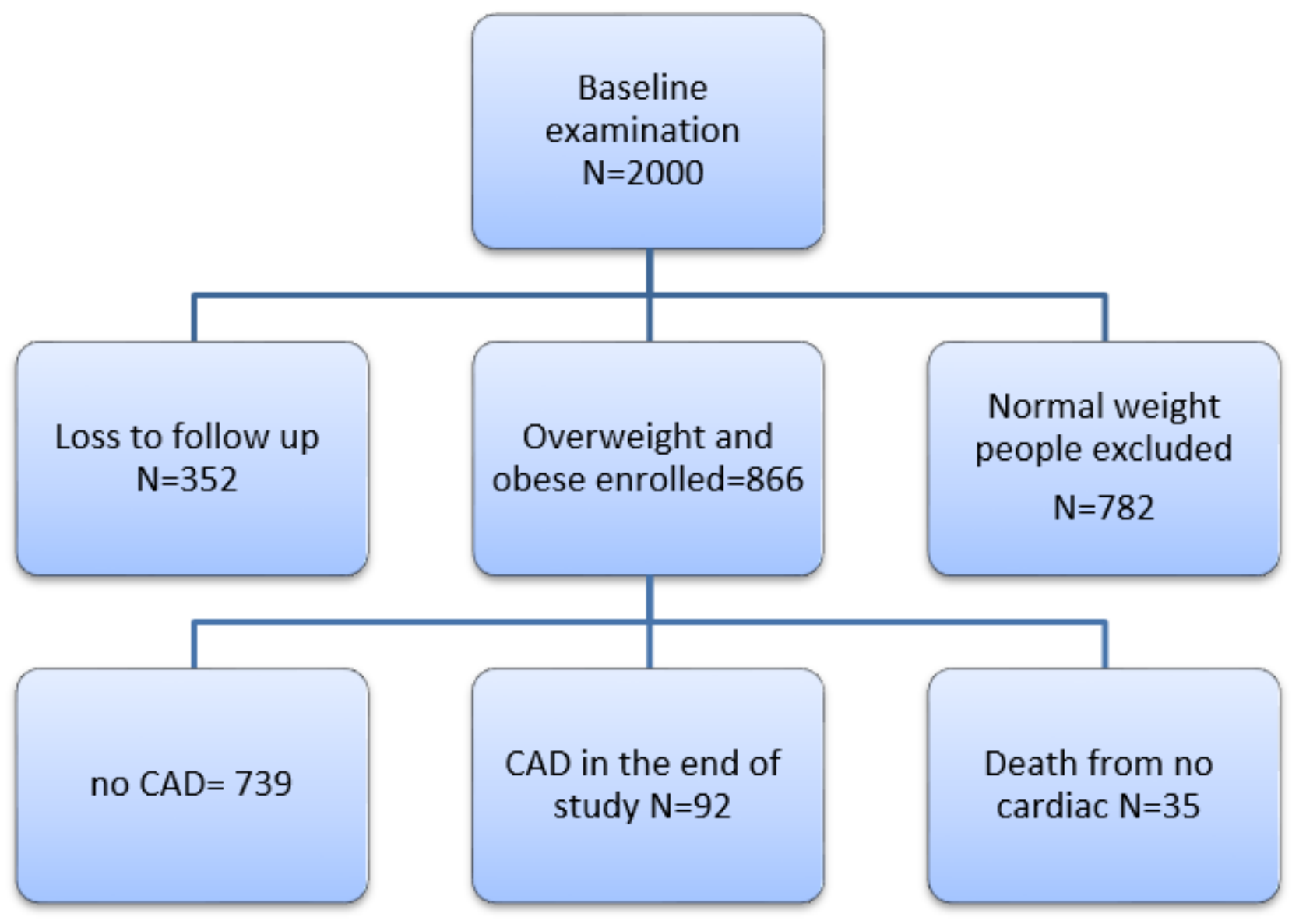

Figure 1

The chart shows population number in baseline and Coronary Artery Disease incidence after 10 years.

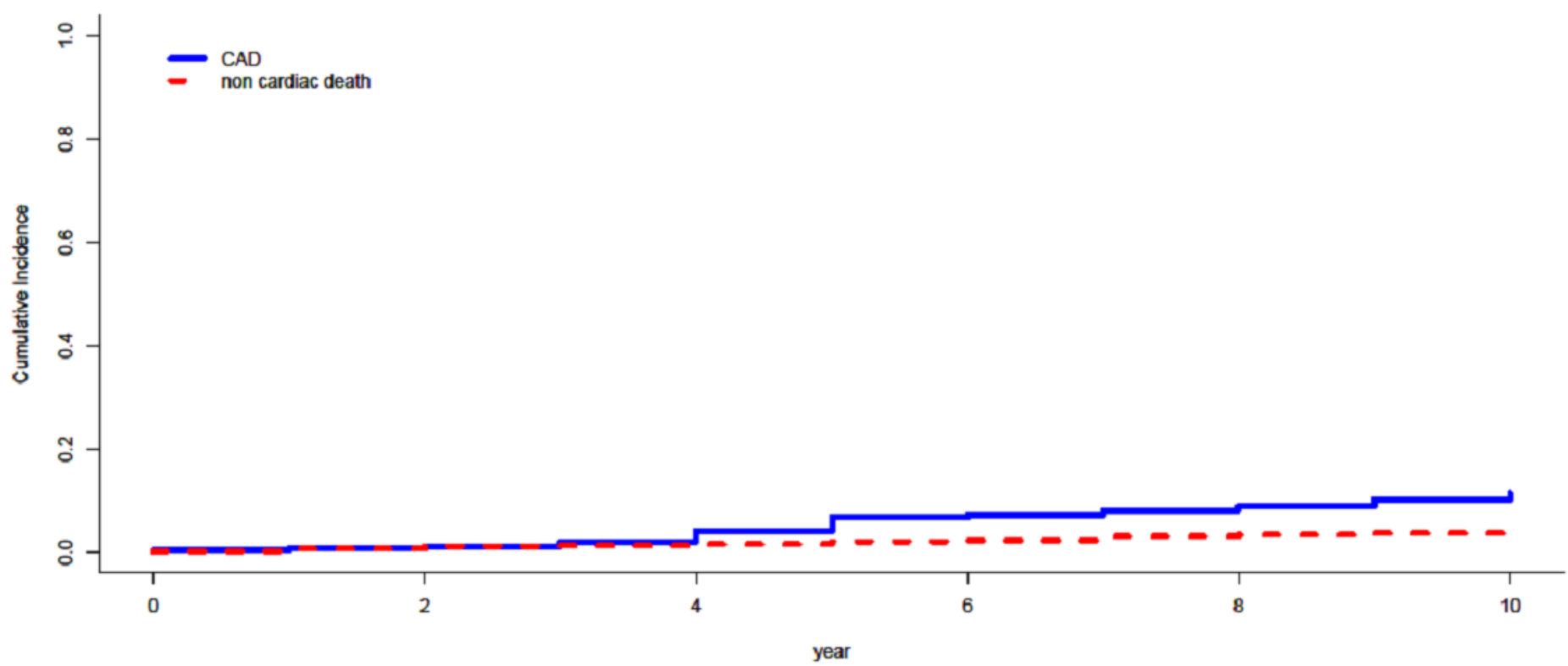


Figure 2

Cumulative Incidence Functions (CIF) curves with $C A D$ incidence as the primary endpoint. $C A D=$ fatal and nonfatal coronary artery disease, all non-cardiac death causes= competing event 